research article

\title{
Numerical modeling in electroporation-based biomedical applications
}

\author{
Nataša Pavšelj and Damijan Miklavčič \\ University of Ljubljana, Faculty of Electrical Engineering, Ljubljana, Slovenia
}

\begin{abstract}
Background. Numerous experiments have to be performed before a biomedical application is put to practical use in clinical environment. As a complementary work to in vitro, in vivo and medical experiments, we can use analytical and numerical models to represent, as realistically as possible, real biological phenomena of, in our case, electroporation. In this way we can evaluate different electrical parameters in advance, such as pulse amplitude, duration, number of pulses, or different electrode geometries. Such numerical models can contribute significantly to the understanding of an experiment and treatment planning as well as to the design of new electroporation devices and electrodes.
\end{abstract}

Methods. We used commercially available modeling software, based on finite element method. We constructed a model of a subcutaneous tumor during electrochemotherapy (EMAS) and a model of skin during gene electrotransfer (COMSOL Multiphysics). Tissue-electrode geometries, pulse parameters and currentvoltage measurements from in vivo experiments were used to develop and validate the models.

Results. To describe adequately our in vivo observations, a tissue conductivity increase during electroporation was included in our numerical models. The output currents of the models were compared to the currents and the voltages measured during in vivo experiments and a good agreement was obtained. Also, when comparing the voltages needed for a successful electropermeabilization as suggested by the models, to voltages applied in experiments and achieving a successful electrochemotherapy or in vivo gene electrotransfer, good agreement can be observed.

Conclusions. Modeling of electric current and electric field distribution during cell and tissue electroporation proves to be helpful in describing different aspects of the process and allowing us to design electrodes and electroporation protocols as a part of treatment planning.

Key words: electroporation; electropermeabilization; electrochemotherapy; subcutaneous tumor; gene electrotransfer; skin; numerical modeling; finite element method

\section{Introduction}

Received 28 June 2008

Accepted 7 August 2008

Correspondence to: Prof. Dr. Damijan Miklavčič, University of Ljubljana, Faculty of Electrical Engineering, Tržaška 25, SI-1000 Ljubljana, Slovenia. Phone: +386 14768 456; Fax: +386 14264 658; E-mail: damijan.miklavcic@fe.uni-lj.si
A cell membrane is, in general, impermeable for molecules; however, the application of electric pulses to cells, either in suspension or in tissue, causes structural changes in the cell membrane. ${ }^{1-3}$ Cell 
membrane is transiently permeabilized due to increased transmembrane voltage caused by external electric field. The phenomenon is also termed electroporation. Even a short electric pulse of a sufficiently high voltage causes an increased permeability of the cell membrane. If the pulse is of adequate amplitude, the electric field and consequently the transmembrane potential are high enough to cause cell membrane permeabilization. The increase in permeability of the cell membrane makes it possible for molecules that otherwise can not cross the membrane, such as drug molecules or DNA, to enter the cell. After exposure to electric pulses, cell membrane reseals provided the applied voltage was not too high to cause permanent damage. Currently, the most widely used applications of electroporation are electrochemotherapy, gene electrotransfer and transdermal drug delivery. The outcome of the electroporation depends on cell and tissue parameters and, most of all, electric pulse parameters.

Electrochemotherapy is one of the most advanced and efficient biomedical applications of electroporation. It is a combination of chemotherapy and electric pulses aimed at temporarily permeabilizing tumor cell membranes to introduce drug molecules more efficiently into the cells. The results of clinical studies show a highly increased efficiency of bleomycin and cisplatin when used in combination with electric pulses. ${ }^{4-6}$ Another promising application of electroporation is gene electrotransfer into cells. It is a method using electric pulses to temporarily and reversibly permeabilize the cell membrane and to drive the DNA into the cell electrophoretically. ${ }^{7}$ This method can be used both in vivo and in vitro and when a transient (e.g. skin $\left.^{8,9}\right)$ or long-term (e.g. muscle $^{10}$ ) transfection is needed. Electroporation can also be used to create aqueous pathways across the skin's outer- most layer, the stratum corneum to enhance transdermal drug delivery. ${ }^{11-13}$

Numerous experiments, both in vitro and in vivo, have to be performed before a biomedical application is put to practical use in clinical environment. As a complementary work to in vivo experimenting, we can use analytical and numerical models to represent, as realistically as possible, real biological phenomena of, in our case, electroporation. ${ }^{14-18}$ In this way we can better understand some of the processes involved and analyze and explain some experimental results. We can evaluate different electrical parameters in advance, such as pulse amplitude, duration, number of pulses. All of that can help us plan new protocols, design electroporation devices, plan new experiments and treatments. Of course, models must always be validated by experiments, and if necessary, improved. Although a model can not replace experimental work entirely, it can show us another aspect of the same problem. Both, experimental work and numerical modeling combined give us valuable information and help us understand the underlying mechanisms. In the present paper, we will show two examples of numerical models of in vivo electroporation; a subcutaneous tumor during electrochemotherapy and skin during gene electrotransfer.

\section{Materials and methods}

Numerical modeling of the electric field and the electric current distributions inside the biological systems represent an important field in the study of the effects of the electromagnetic fields on cells, tissues and organs. It is a relatively simple yet powerful tool for analysis and explanation of intricate processes taking place inside biological systems. Various electrical parameters (current and voltage amplitude, field strength and 
orientation, electrode geometries...) can be evaluated by means of numerical modeling. Namely, experimenting with such models is easier and sometimes the only possible or ethically acceptable alternative to experimenting on real biological systems. Our models are based on the finite elements method (FEM). The essence of the method is the discretization of the geometry into smaller elements - finite elements - where the quantity of interest is approximated with a simple function. Mathematically, the finite element method is used for finding an approximate solution of partial differential equations (PDE). For our work, we were using commercially available modeling software EMAS (Ansoft, Pittsburgh, PA, USA) - for the tumor model and COMSOL Multiphysics, (COMSOL, Los Angeles, CA, USA) for the skin fold model, both based on finite element method.

\section{Theoretical background}

The bulk properties of biological materials are important in many applied problems of electrical stimulation. They dictate the current densities and pathways that result from an applied stimulus and are thus very important in the analysis of a wide range of biomedical applications. ${ }^{19}$ To analyze the response of a tissue to electric excitation with direct current, we need data on the conductivities of the tissues or organs. Electrical conductivity is a measure of a material's ability to conduct electric current. When an electrical potential difference exists on a conductor, its free charges start moving, which results in an electric current. Electrical conductivity $(\sigma)$ is defined as the ratio of the current density to the electric field strength $(\sigma=J / E)$ and has the units of Siemens per meter $(\mathrm{S} / \mathrm{m})$. Material's ability to conduct electric current can also be given by its electrical resistivity $(\rho)$. Electrical resistivity is the inverse of the electrical con- ductivity and is a measure of how strongly a material opposes to the flow of electric current. A low resistivity indicates a material that readily allows the movement of electrical charge. The unit of electrical resistivity is the Ohm meter $(\Omega \mathrm{m})$.

It is very important not to confuse the electrical conductivity and the electrical resistivity with the conductance and the resistance. Similarly to the definitions above, we can state that electrical conductance $(G)$ is a measure of an object's (not material's) ability to conduct electric current, and electrical resistance $(R)$ is a measure of how strongly an object (not a material) opposes to the flow of electric current. An object's electrical conductance (electrical resistance) is thus a function of both its physical geometry and the conductivity (resistivity) of the material it is made from:

$$
\begin{aligned}
& G=\sigma A / l \\
& R=\rho l / A
\end{aligned}
$$

Where $l$ is the object's length, $A$ is its cross sectional area, $\sigma$ and $\rho$ are the conductivity and the resistivity of the material, respectively.

If we know the electric potential difference (the voltage $U$ ) and the electrical conductance (or electrical resistance) of the object, we can calculate the electric current $I$ :

$$
\begin{aligned}
& I=G \cdot U \\
& I=U / R
\end{aligned}
$$

Upon applying electric pulses on a setup of more materials (tissues) with different dimensions and electrical conductivities (electrical resistivities), connected in a serial configuration (e.g. skin), the voltage is divided among them proportionally to their electrical resistances, as in a voltage divider represented in Figure 1a. Similarly, where 


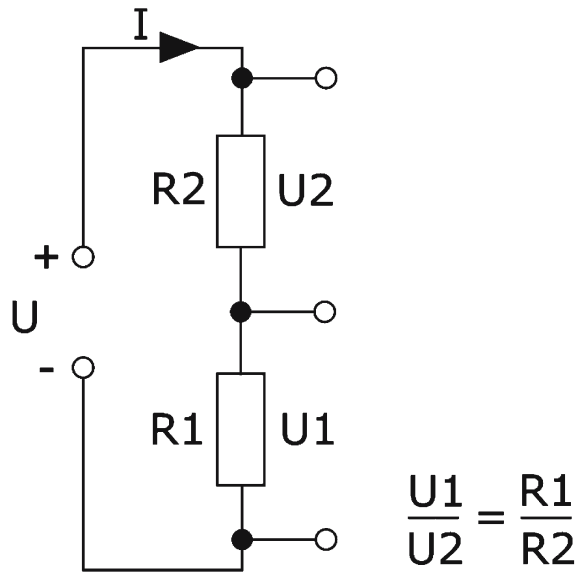

a)

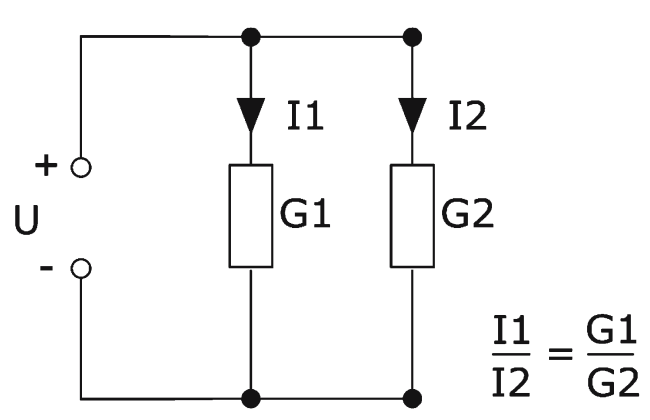

b)

Figure 1. a) voltage divider, b) electric current divider

objects of different electrical conductances are in parallel configuration, the current is divided among them proportionally to their electrical conductances, as in a current divider represented in Figure 1b.

\section{Numerical models - geometry}

Experimental results show a successful electrochemotherapy of a subcutaneous tumor when pulses are delivered through external plate electrodes. ${ }^{4}$ Also, a successful

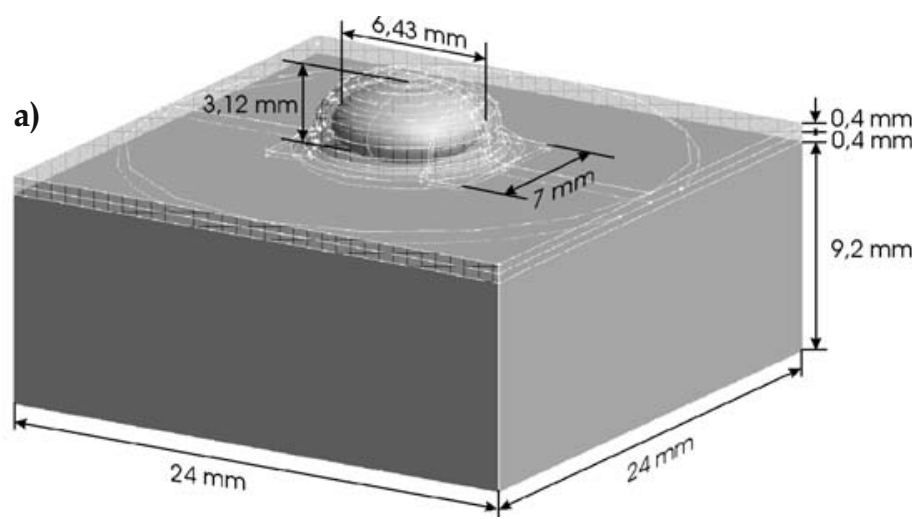

gene electrotransfer into rat skin cells was achieved when skin fold was formed and placed between plate electrodes delivering electric pulses. ${ }^{9}$ Numerical models were made in order to describe theoretically the

Figure 2. Geometries of finite element numerical models. a) The subcutaneous tumor model made in EMAS. b) One quarter of the skin fold model made in COMSOL. 


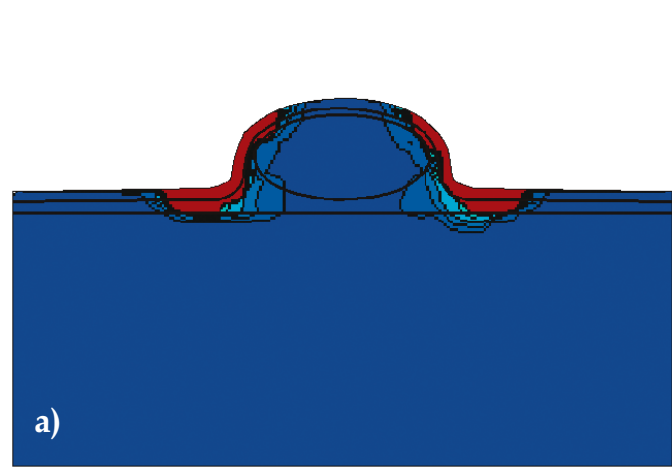

Figure 3. The electric field distributions before tissue permeabilization; a) The subcutaneous tumor model, results are shown in a section plane cut through the middle of the model, perpendicular to electrodes. b) The skin fold model, results are shown in 5 section planes cut perpendicularly to electrodes. The electric field is shown in volts per centimeter.

process of tissue electropermeabilization. Tissue-electrode geometries, pulse parameters and current-voltage measurements from in vivo experiments were used to develop the models. The geometries of both models are shown in Figure 2 and were made as close to the in vivo experimental tissue-electrode set-ups as possible. In the case of the subcutaneous tumor four different tissues were modeled: skin, subcutaneous fat, tumor and the underlying muscle. ${ }^{18}$ In the case of the skin fold, skin's layered structure was modeled: stratum corneum, epidermis, dermis and the subcutaneous layer of fat and connective tissue. ${ }^{16}$

\section{Numerical models - the electroporation process}

In tissue, the voltage is the highest in the layer with the highest resistivity (the lowest conductivity). This leads to a certain electric field distribution (as in voltage divider), meaning that different layers are exposed to different electric field strengths. The electric field is the highest in the layer with the highest resistivity (lowest conductivity).
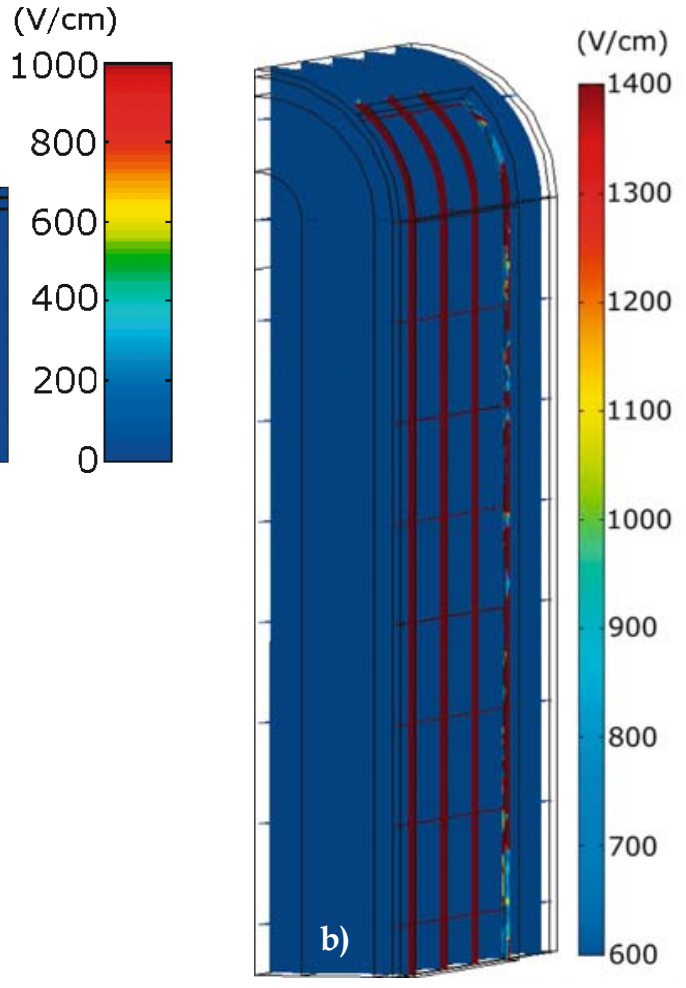

In the case of the subcutaneous tumor this is the skin, which has the lowest electrical conductivity, and in the case of the skin fold, the highest electric field is in the non-conductive outermost skin layer, the stratum corneum. This can be clearly seen in Figure 3, showing the electric field distribution in the two models at $1000 \mathrm{~V}$ and 400 $\mathrm{V}$ between the plates, respectively. We can observe a very high electric field in the tissues with the highest electrical resistivity, while the electric field in the target tissues (tumor and viable skin layers) stays too low for successful electropermeabilization.

This fact raises the question of how is the experimentally confirmed successful permeabilization of the target tissues theoretically possible when external plate electrodes are used. The answer lies in the increase in bulk conductivities of the permeabilized tissues, a phenomenon that 

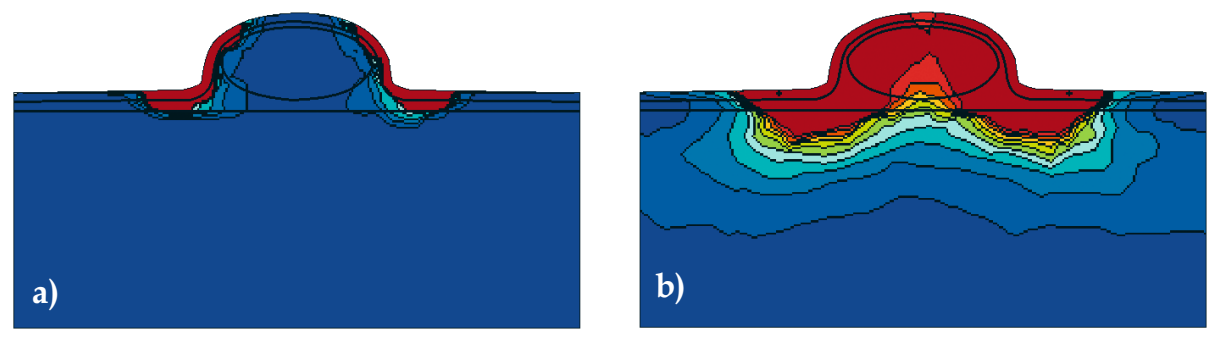

$E(V / c m)$
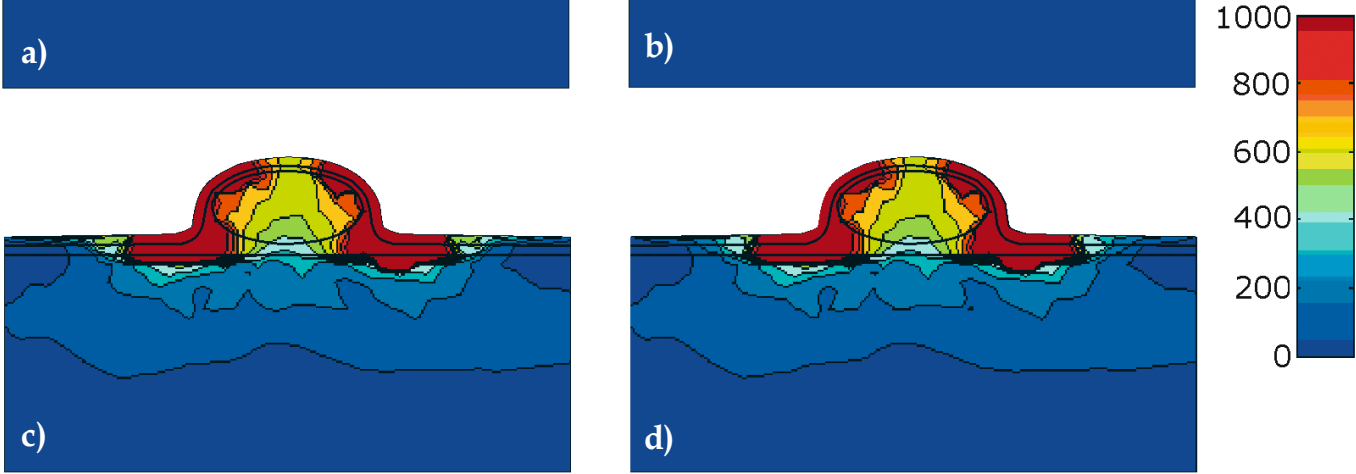

Figure 4. Electric field distributions in subcutaneous tumor, results are shown in a section plane cut through the middle of the model, perpendicular to electrodes for $1000 \mathrm{~V}$ between two external plate electrodes of $8 \mathrm{~mm}$ distance. Electric field distributions are shown in four time steps, from the non-permeabilized state (a), to the tissues being fully permeabilized (d). The electric field distribution is shown in $\mathrm{V} / \mathrm{cm}$.

was also observed in vivo. Namely, the high electric field in skin / stratum corneum is above the permeabilization threshold, which causes the electropermeabilization of the two tissues. As a consequence, the conductivity of skin / stratum corneum increases, and the electric field distribution is changed. In this way, the electric field high enough reaches the target tissues below skin and the stratum corneum. Therefore, our numerical models have to reflect this nonlinear dependence of conductivity on electric field. The electric field distribution (the model output) depends on the changes in the electrical conductivity of the tissues involved (model input parameters), the numerical analysis needs to be performed in iterations.

Table 1. Conductivity values used for tissues/skin layers represented in our models, where $\sigma_{0}$ denotes initial tissue conductivity, and $\sigma_{1}$ is the conductivity of permeabilized tissue.

\begin{tabular}{l|l|l|l}
\hline & Tissue & $\sigma_{\mathbf{0}} \mathbf{( S / m )}$ & $\sigma_{\mathbf{1}}(\mathbf{S} / \mathbf{m})$ \\
\hline \multirow{3}{*}{ Subcutaneous tumor } & $\begin{array}{l}\text { Subcutaneous layer } \\
\text { Skeletal muscle } \\
\text { (longitudinal/transverse) } \\
\text { Tumor }\end{array}$ & 0.03 & 0.09 \\
& Skin & $0.735 / 0.11$ & $2.94 / 0.44$ \\
\hline \multirow{3}{*}{ Skin fold } & Subcutaneous layer & 0.002 & 0.8 \\
& Dermis, viable epidermis & 0.05 & 0.16 \\
\hline & Stratum corneum & 0.2 & 0.2 \\
\hline
\end{tabular}



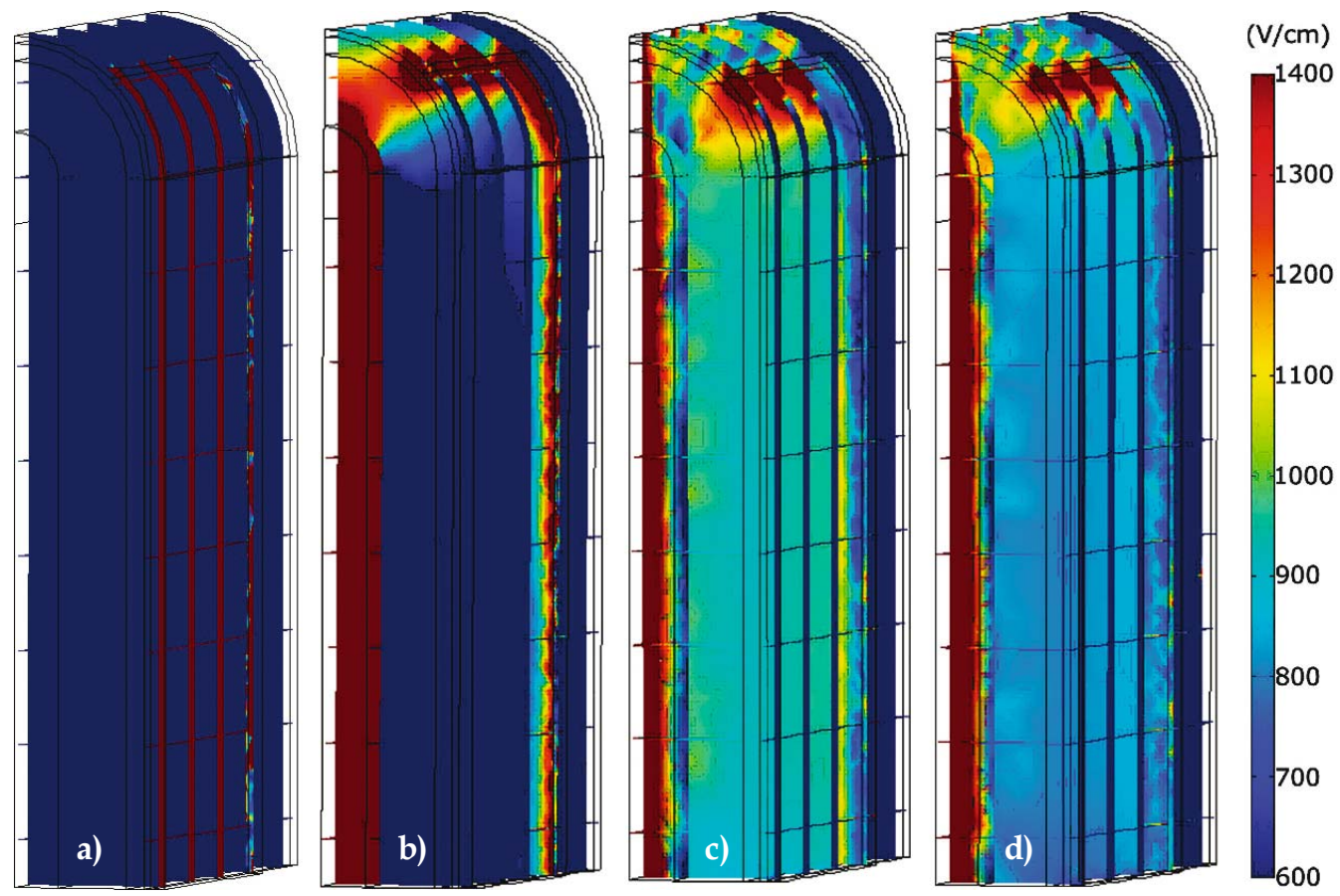

Figure 5. Three dimensional slice plots of the electric field distributions during the electropermeabilization process in the skin fold for the applied voltage $400 \mathrm{~V}$ between two plate electrodes of $4 \mathrm{~mm}$ distance. Electric field distributions are shown in four time steps, from the non-permeabilized state (a), to the skin layers being fully permeabilized (d). The electric field distribution is shown in V/cm.

Exactly how tissue conductivities change with electric field is another unknown or poorly known parameter. By using our own experiments and literature data ${ }^{20-35}$, we set the initial and the permeabilized conductivity values of the tissues in both models as given in Table 1.

\section{Results and discussion}

The improved, nonlinear models where tissue conductivities change according to the current electric field were solved for different electric pulse amplitudes. The subcutaneous tumor model was solved for $500 \mathrm{~V}, 1000 \mathrm{~V}$ and $1500 \mathrm{~V}$, while the skin model was solved for $160 \mathrm{~V}, 280 \mathrm{~V}, 400 \mathrm{~V}$, $520 \mathrm{~V}$ and $700 \mathrm{~V}$.
For the case of the subcutaneous tumor, the electric field distributions at $1000 \mathrm{~V}$ are shown at 4 time steps (Figure 4). The first step is the same as shown before (Figure 3a), with the highest electric field in the skin, while the electric field in other tissues is very low. But due to the conductivity changes of the permeabilized tissues, the next time step shows a different picture. The high electric field reaches the tissues below, permeabilizing them, thus changing the electric field distribution again. This process is repeated until we reach the steady state. The last step shows a high enough electric field for its permeabilization throughout the tumor, which is what we also observed in vivo - a successful electrochemotherapy at this voltage and electrode-tissue geometry. 

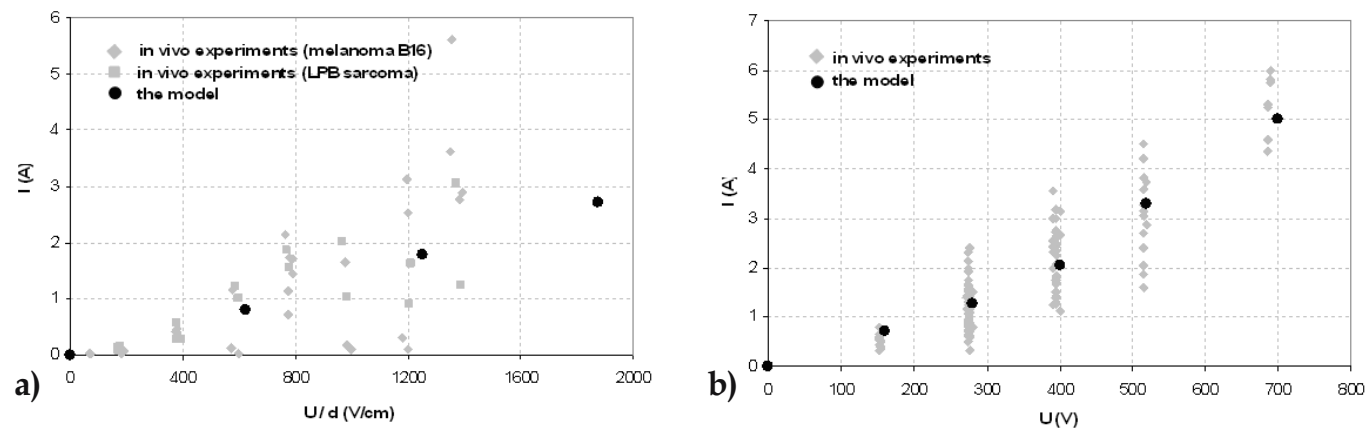

Figure 6. Currents measured during the pulse, compared to the currents given by the models, with respect to applied voltages for a) Subcutaneous tumor: The distance between plate electrodes was not uniform, hence the applied voltage is given in volts per centimeter; b) Skin fold

Further, the electric field distribution in skin fold shows similar progression (Figure 5). The first step is again the same as shown in Figure $3 b$, with the highest electric field in the stratum corneum, and a very low electric field in the target tissues below. Again, due to the conductivity changes, a high enough electric field moves to the tissues below stratum corneum, thus permeabilizing the viable epidermis and the dermis. Again, this agrees with our in vivo results. Namely, a high-level gene expression was observed at this voltage.

For further comparison of the models with the in vivo results the currents computed with the model were compared to the stationary currents flowing through the tissue, measured in vivo during the pulse (Figure 6). A good agreement can be observed for both models. Further, the current/voltage dependence given by our models exerts the nonlinearity observed in the in vivo data, suggesting that the approach we used to describe the process explains well the nonlinear nature of tissue electropermeabilization.

\section{Conclusions}

Numerical modeling of the electric field and the electric current distributions in- side the biological systems represent an important field in the study of the effects of the electromagnetic fields on cells, tissues and organs. It is a powerful tool for the analysis of various electrical parameters and the explanation of the intricate processes taking place inside the biological systems. We have shown examples of numerical modeling on two electroporation-based applications: electrochemotherapy of subcutaneous tumors and skin gene electrotransfer. The regression of tumor growth after electrochemotherapy, proven also in clinical environments, and a successful gene electrotransfer to skin cells had shown that deeper target tissues (tumor, the dermis and the viable epidermis) can be permeabilized when external plate electrodes are used. The electropermeabilization of these tissues was possible even though the ratios of the nonpermeabilized tissue conductivities suggest that the electric field in the target tissues will be too low for a successful electropermeabilization. However, a phenomenon we can observe in the in vivo experiments is the increase in tissue conductivity due to cell membrane electroporation. This conductivity increase of the permeabilized tissues was included in our numerical models. The output currents of the models were compared to the stationary currents and the voltages 
measured during in vivo experiments and a good agreement was obtained. Also, based on already published in vivo experiments and comparing the voltages needed for a successful electropermeabilization as suggested by the models, with voltages achieving a successful electrochemotherapy or in vivo gene electrotransfer, good agreement can be observed. ${ }^{4,9,16}$

In conclusion, with the models presented in this paper we used the available data in order to explain the mechanism of tissue electropermeabilization. Our models serve as a proof of principle and proved useful for describing different aspects of the observed process. Furthermore, such numerical models can help us design electrode geometries and electroporation protocols as a part of treatment planning.

\section{Acknowledgements}

This research was supported by the European Commission and the Slovenian Research Agency.

\section{References}

1. Tsong TY. Electroporation of cell membranes. Biophys J 1991; 60: 297-306.

2. Prausnitz MR, Corbett JD, Gimm JA, Golan DE, Langer R, Weaver JC. Millisecond measurement of transport during and after an electroporation pulse. Biophys J 1995; 68: 1864-70.

3. Teissié J, Eynard B, Gabriel B, Rols MP. Electropermeabilization of cell membranes. Adv Drug Deliver Rev 1999; 35: 3-19.

4. Sersa G, Cemazar M, Miklavcic D. Antitumor effectiveness of electrochemotherapy with cis-diamminedichloroplatinium (II) in mice. Cancer Res 1995; 55: 3450-5.

5. Heller R, Gilbert R, Jaroszeski MJ. Clinical application of electrochemotherapy. Adv Drug Deliver Rev 1999; 35: 119-29.
6. Sersa G, Cemazar M, Miklavcic D, Rudolf Z. Electrochemotherapy of tumours. Radiol Oncol 2006; 40: 163-74

7. Hojman P, Gissel H, Gehl J. Sensitive and precise regulation of haemoglobin after gene transfer of erythropoietin to muscle tissue using electroporation. Gene Therapy 2007; 14: 950-9.

8. Zhang L, Nolan E, Kreitschitz S, Rabussay DP. Enhanced delivery of naked DNA to the skin by non-invasive in vivo electroporation. Biochim Biophys Acta 2002; 1572: 1-9.

9. Pavselj N, Préat V. DNA electrotransfer into the skin using a combination of one high- and one low-voltage pulse. J Control Release 2005; 106: 40715.

10. Mir LM, Bureau MF, Gehl J, Rangara R, Rouy D, Caillaud J-M, et al. High-efficiency gene transfer into skeletal muscle mediated by electric pulses. PNAS 1999; 96: 4262-7.

11. Prausnitz MR. The effects of electric current applied to skin: A review for transdermal drug delivery. Adv Drug Deliver Rev 1996; 18: 395-425.

12. Prausnitz MR. A practical assessment of transdermal drug delivery by skin electroporation. Adv Drug Deliver Rev 1999; 35: 61-76.

13. Denet A-R, Vanbever R, Préat V. Skin electroporation for transdermal and topical delivery. Adv Drug Deliver Rev 2004; 56: 659-74.

14. Dev SB, Dhar D, Krassowska W. Electric field of a six-needle array electrode used in drug and DNA delivery in vivo: Analytical versus numerical solution. IEEE T Bio-Med-Eng 2003; 50:1296-300.

15. Zupanic A, Corovic S, Miklavcic D. Optimization of electrode position and electric pulse amplitude in electrochemotherapy. Radiol Oncol 2008; 42: 93101.

16. Pavselj N, Préat V, Miklavcic D. A numerical model of skin electropermeabilization based on in vivo experiments. Ann Biomed Eng 2007; 35: 2138-44.

17. Sel D, Macek-Lebar A, Miklavcic D. Feasibility of employing model-based optimization of pulse amplitude and electrode distance for effective tumor electropermeabilization. IEEE T Bio-Med-Eng 2007; 54: 773-81.

18. Pavselj N, Bregar Z, Cukjati D, Batiuskaite D, Mir LM, Miklavcic D. The course of tissue permeabilization studied on a mathematical model of a subcutaneous tumor in small animals. IEEE T BioMed-Eng 2005; 52: 1373-81. 
19. Miklavcic D, Pavselj N, Hart FX. Electric properties of tissues. In: Wiley encyclopedia of biomedical engineering. New York: John Wiley \& Sons; 2006. p. 3578-89.

20. Pliquett $U$, Langer $R$, Weaver JC. Changes in the passive electrical properties of human stratum corneum due to electroporation. Biochim Biophys Acta 1995; 1239: 111-21.

21. Schmeer M, Seipp T, Pliquett U, Kakorin S, Neumann E. Mechanism for the conductivity changes caused by membrane electroporation of CHO cell-pellets. Phys Chem Chem Phys 2004; 6: 5564-74.

22. Geddes LA, Baker LE. The specific resistance of biological material-a compendium of data for the biomedical engineer and physiologist. Med Biol Eng 1967; 5: 271-93.

23. Schwan HP, Kay CF. Specific resistance of body tissues. Circ Res 1956; 4: 664-70.

24. Epstein BR, Foster KR. Anisotropy in the dielectric properties of skeletal muscle. Med Biol Eng Comput 1983; 21: 51-5.

25. Burger HC, Van Dongen R. Specific resistance of body tissues. Phys Med Biol 1960; 5: 431-447.

26. Rush S, Abildskov JA, McFee R. Resistivity of body tissues at low frequencies. Circ Res 1963; 12: 40-50.

27. Gabriel C, Gabriel S, Corthout E. The dielectric properties of biological tissue: I. Literature survey. Phys Med Biol 1996; 41: 2231-49.

28. Gabriel S, Lau RW, Gabriel C. The dielectric properties of biological tissue: II. Measurements in the frequency range $10 \mathrm{~Hz}$ to $20 \mathrm{GHz}$. Phys Med Biol 1996; 41: 2251-69.

29. Smith SR, Foster KR, Wolf JL. Dielectric properties of VX-2 carcinoma vs. normal liver tissues. IEEE T Bio-Med-Eng 1986; 33: 522-4.

30. Surowiec AJ, Stuchly SS, Barr JR, Swarup A. Dielectric properties of breast carcinoma and the surrounding tissues. IEEE T Bio-Med-Eng 1988; 35: 257-63.

31. Gielen FLH, Wallinga-de Jonge W, Boon KL. Electrical conductivity of skeletal muscle tissue: Experimental results from different muscles in vivo. Med Biol Eng 1984; 22: 569-77.

32. Yamamoto T, Yamamoto Y. Electrical properties of the epidermal stratum corneum. Med Biol Eng 1976; 14: 151-58.
33. Yamamoto T, Yamamoto Y. Dielectric constant and resistivity of epidermal stratum corneum. Med Biol Eng 1976; 14: 494-500.

34. Chizmadzhev YA, Indenbom AV, Kuzmin PI, Galichenko SV, Weaver JC, Potts RO. Electrical properties of skin at moderate voltages: Contribution of appendageal macropores. Biophys J 1998; 74: 843-56.

35. Gallo SA, Oseroff AR, Johnson PG, Hui SW. Characterization of electric-pulse-induced permeabilization of porcine skin using surface electrodes. Biophys J 1997; 72: 2805-11. 